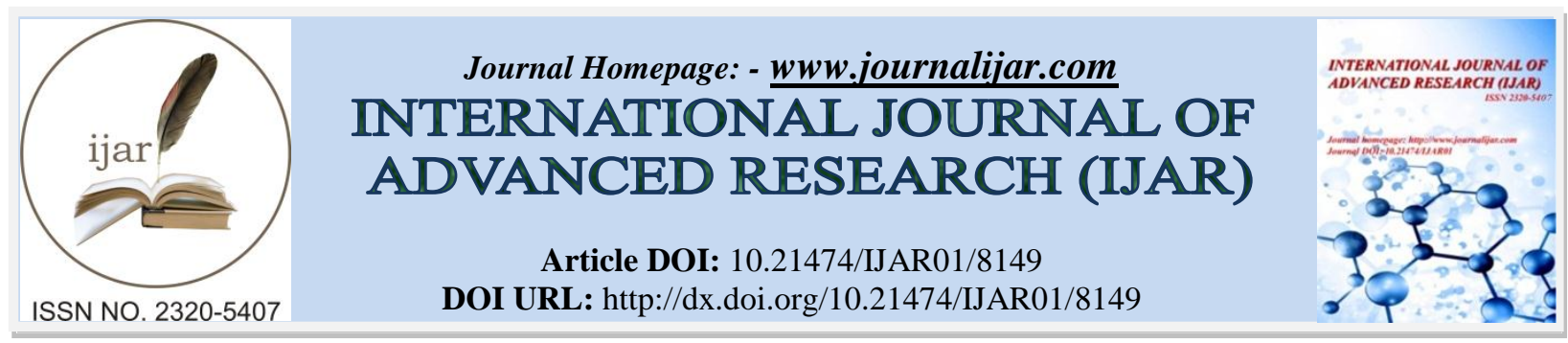

RESEARCH ARTICLE

\title{
REAL-TIME REMOTE MONITORING URBAN AND ARCHITECTURAL 3D DIGITAL DESIGN AND SPATIAL SIMULTANEOUS SIMULATION.
}

Stelios Kouzeleas.

Laboratory of rural area, Department of Planning \& Regional Development, University of Thessaly, Greece.

\section{Manuscript Info}

\section{Manuscript History}

Received: 04 October 2018

Final Accepted: 06 November 2018

Published: December 2018

Keywords:

Real-time digital design and spatial simulation (digital representations), CAD, 3D modeling, Spatial Real-time

Remote Monitoring Systems,

Computational design.

\section{Abstract}

Object: The present research takes advantage of wide spread technologies such as GPS, VHF telecommunications, field sensors and techniques commonly used in telegeoprocessing and teleomonitoring procedures in order to remotely model and simulate an existing urban or architectural environment with its spatial properties such as sound, temperature, humidity, etc. It also presents and compares adequate systems using almost similar technologies which contribute to a remote spatial urban simulation.

Methods: This research describes a method process of a remote system that captures and transmits spatial data collected from the real urban or architectural environment directly, in real-time, into a CAD 3D modeling and simulation environment. These spatial data are manipulated adequately via specially developed interfaces in order to be simulated simultaneously in superposed layers cartography.

Results: The remote feed of CAD software with spatial data provokes the drawing of new 3D real entities in architectural scale (rooms, squares, buildings position, roads, etc.), or in urban scale (relief, morphology, etc.) in real-time, as the field-user handles a peripheral device of the modelling software (which is connected wirelessly), creating automatically a superposed cartography of spatial properties (sound pressure level, intensity, noise, temperature, etc.). This methodology was applied to the Diachronic Museum of Larissa Greece by creating a relief of its surround area, its buildings positions and a superposed sound pressure level cartography.

Conclusions: Each entity, which is created in real-time, can directly participate in any spatial analysis operation without further treatment. The space and its properties simulation can constitute a common base of holistic description of space with huge possibilities of development and applications.

Copy Right, IJAR, 2018,. All rights reserved.

\section{Introduction:-}

Taking into account that an "active" space is changing continuously, as various events are happening, there is a need to monitor space through time using real-time models. Contemporary techniques of geosciences involving telecommunication means facilitate data transfer processing and simulation directly from the field to a computer

Corresponding Author:- Stelios Kouzeleas. 
system of modeling or simulation environment contributing to the emergence of a new concept called "telegeomatic" (Laurini, 2000). The telegeomatic technology is a combination of spatial decision support systems (DSS) in real-time context, geographical information systems (GIS) or design systems (CAD) and communication processes such as open communication systems (OCS) integrating new information and communication technologies such as Internet, mobile and wireless applications (Boulmakoul, 2006).

These techniques are more and more applied in many engineering processes and fields, such as meteorology, survey, urbanism, urban planning and design, acoustics, etc., which require data collection (field survey), adequate processing (Albrecht, 1996) and final real-time simulation. In the majority of cases, the results are simulated in two ways:

1. in "traditional" unique layer cartography representing either a geographical map with dynamic features with Geographical Information System (Weis et al, 2005) focuses on geomorphologic aspects of space representation,

2. in spatial property cartography (e.g., sound, humidity, temperature, etc.) referring to spatial geographical coordinates,

(Papadimitriou and Kouzeleas, 2005).

In the first case, the cartography focuses on the geographical representation of space involving approaches in GIS for coordinate's accuracy, measurements of spatial data quality, measuring positional error for points, lines and polygons, modeling attribute and temporal uncertainties (Devillers et al, 2010). At the same time, different similar approaches concern remote sensing techniques looking at various quality aspects of images from the collection to the processing and analysis of the images; thus, generated systems can aid in updating existing GIS data by interpreting images automatically where the verification of GIS objects from images is performed by these systems which introduce GIS information into the knowledge base for image processing (Weis et al, 2005).

In the second case, unique or multi layer cartography georeferences spatial property as a crucial characteristic of space perception using also remote monitoring and creating real-time applications. These techniques use digital, multiple networks of sensors or unique sensors (Goni et al, 2009) in order to capture, and finally simulate spatial property measurements following a specific process in each case which influences significantly the final simulation results in a multitude of applications fields.

Contemporary systems such as telegeoprocessing and telegeomonitoring systems allow data transfer from a recording device to a processing system; their basic components are the telecommunications, the GPS system and the spatial data management systems such as GIS, CAD, etc. In that kind of array, the GPS devices are exploited for the position location and then the telecommunication means is also exploited to send the coordinates, sometimes with descriptive information, to a computer for their processing.

This study attempts to present 3D remote digital design and simulation systems of space description and also to combine theses two ways of geomorphologic and spatial properties simulation by describing a methodology of spatial design and simulation process using telemonitoring techniques. This methodology concerns a remote sending of data combination of position, time and captured rates by a digital mobile sensor. The suggested system records coordinates in real-time from the real space in order to generate automatically points, linear or surface models in a modelling environment such as AutoCAD. It is about a low cost, but very efficient, mobile devices array which is used for measurements capture describing selected properties of space which are transmitted in real-time from the field towards a system which models and simulates in 3D the space and its georeferred properties while repeated measurements are refreshing the model. This imposes the interoperability (OGC, 2010) between systems with different origins (e.g. GIS, telecommunications, digital sensors, etc). Those are particularly useful for remote monitoring and real-time applications (Tanzi, 2000). In such cases, it takes time to perform surveying and processing sequentially. Assuming that the time for the field survey (data capture) cannot be avoided, one can take advantage of that time and simultaneously process the captured data within a modelling or simulation environment such as a CAD system. Thus, the automation of the:

1. transmitted data elaboration so as to be adequate in a $\mathrm{CAD}$ environment,

2. modeling operations and

3. solutions of different nature problems such as interfaces compatibility, models entities simplification, simulation accuracy, etc, are very important in order to structure an adequate methodology of real-time remote digital design and simulation. 
The final goal of the proposed methodology is the holistic approach, simulation and understanding of space via a multitude of complementary spatial properties cartography creation in simultaneous superimposed simulation with geographic reference to the existent cartography of geomorphologic features. The proposal concerns a research work in theoretical, for instance, development in the phase before its application in adequate areas of interest and for this reason, on the one hand, specific cases of application of the proposed methodology are not presented yet and, on the other hand, a detailed analysis of developed algorithms, interfaces programming development and technical modeling operations is intentionally avoided in this paper.

\section{A brief retrospective of remote monitoring:-}

The digital era arrived in 1972 with the launch of satellite Landsat 1 by the National Aeronautics and Space Administration (NASA) which carried a television camera, a satellite-relay system for land-based telemetry and electronic sensors that could make pictures with colors. A Tracking and Data Relay Satellite (TDRS) is a type of communications satellite which is an artificial satellite stationed in space for the purpose of telecommunications used by NASA (Schneider and Garman, 1981). Special areas were monitored for many months using Landsat-1 to transmit data successfully every few minutes intervals while these data were generally available within $12 \mathrm{hrs}$ of transmission from the field site (Glasgow et al, 2004). However, only $2 \%$ of all data observations were successfully relayed due to different problems such as satellite orbit effects, hardware malfunction, overloading of recording devices, battery failure, etc. (Paulson, 1975; Turner and Woodham, 1980). In the same year (1972), the United States Geological Survey (USGS) began experimenting using satellite-relay systems included significantly reduced manpower resources to operate large data-collection networks and automatically process data (Glasgow et al, 2004). Since 1972, six other satellites were launched with multi-spectral scanners, carrying new more advanced sensors and providing greater resolution and valuable information as well as detailed observations about the surface of the Earth (Glasgow et al, 2004). The latest generation remote sensing satellite, which was launched in 1999 and it is a joint initiative of the USGS and NASA, has the mission to establish and execute a data acquisition strategy that ensures repetitive acquisition of observations over the Earth's land mass, coastal boundaries, and coral reefs; and to ensure the data acquired are of maximum utility in supporting the scientific objectives of monitoring changes in the Earth's land surface and associated environment (Netting, 2010). This satellite launching initiates the Landsat-7 Long-Term Acquisition Plan (LTAP) which is an important step forward in the long series of Earth observations currently archived by the USGS (Short et al, 1976). The core of the LTAP is the definition of seasonality for each scene of interest, where seasonality is defined as the occurrence of change over time. When significant change occurs, acquisition frequencies are high and conversely, if little change occurs, acquisition frequencies are low. The basic unit of acquisition is a "scene," a segment of the sensor's 183 -km-wide swath extending laterally over $170 \mathrm{kms}$ $(31,000 \mathrm{~km} 2)$. In only the first two years of functioning a database of just over 14,000 scenes containing "land" was compiled for the LTAP (Arvidson, 2001). Since then, a thousand of applications have been performed with Landsat data to meet the needs of business, government, science and education, such as water resource estimates, geological surveys, forest management inventories, agricultural evaluations, land changes, etc (Fig. 1) (Nature, 2013). The experience of the Landsat programs has confirmed the importance of non-sensor specific program characteristics including a global data acquisition strategy, easy data access, low cost availability, long-term data archiving, and web based ordering and delivery (Wulder et al, 2008).

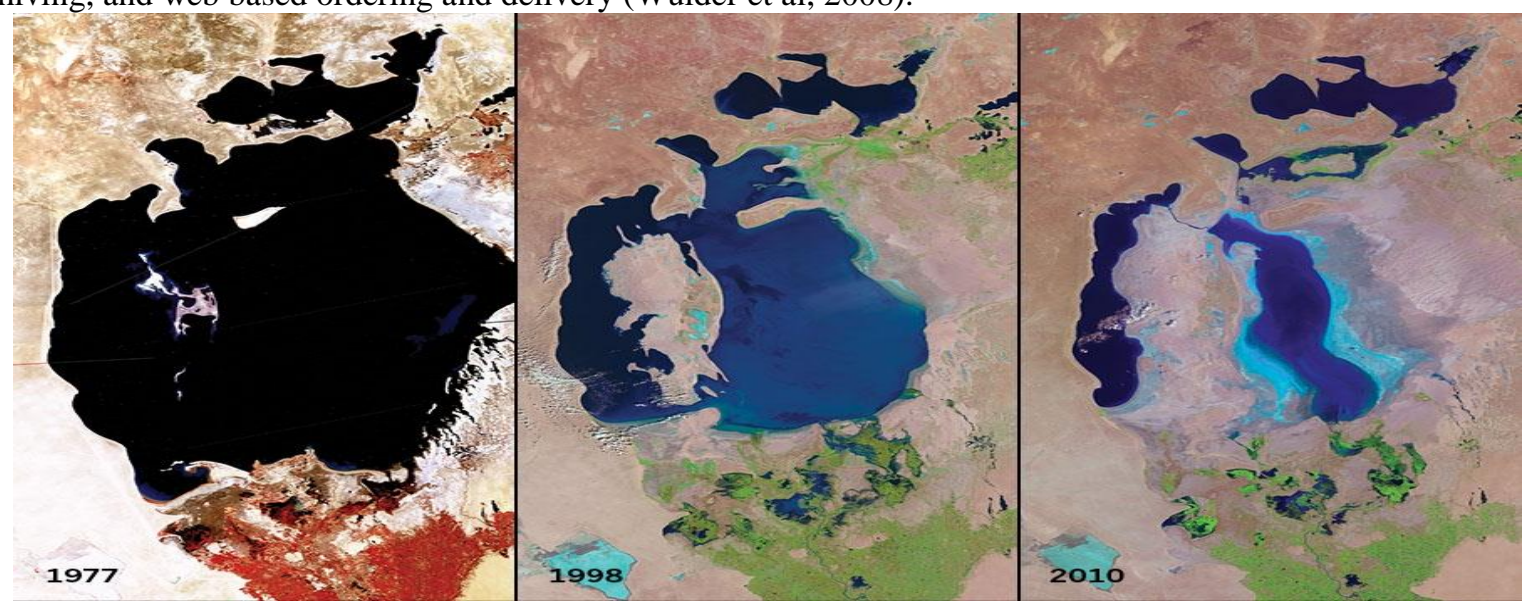

Fig 1:-Landsat satellite data, acquired and archived by the U. S. Geological Survey over the decades, are used by resource managers to measure the Aral Sea's extent and rate of drying. Each Landsat scene covers an area $170 \mathrm{~km}$ 
north/south x $185 \mathrm{~km}$ east/west (106 miles x 115 miles). Images from the Landsat satellite series show the Aral Sea in central Asia shrinking significantly from 1977 to 2010 because of water diversion for agricultural use

(Nature, 2013).

The first geostationary weather satellite, the U.S. Geostationary Operational Environmental Satellite (GOES-1) was launched in 1975 and was deactivated by NASA in March 1985 after 10 years of service providing precious information in the National Weather Service operations (Glasgow, 2004). Since then, new satellite generation was launched, from GOES-9 to 12 with sensors to measure Earth-emitted and reflected radiation from which atmospheric temperature, fire detection, winds, moisture and cloud cover can be derived (Xu et al, 2010).

During the last thirty years, new operational and forthcoming technologies of new satellites have been developed. The use of remote sensing data, whether air-, satellite- or ground-based in relation with the very high spatial resolution optical and infrared land space imagery of Quickbird, Ikonos, CartoSat-1, IRS, ALOS, SAR, etc, provides high level of detection and identification data, monitoring and finally spatial analysis and hazard prediction (Metternicht et al, 2005). These transmission data which are collected by local ground stations and other sites, such as USGS, etc, are updated to the Word Wide Web in a total time of usually 7-8 min using computer technologies. The development of web-based services permits the exploitation of the Real-Time Remote Monitoring systems (RTRM) combining the virtual geography as the cyberspace, which is defined as the space inside computers that changing material place outside computers (Batty, 1997), and the material real space, creating an hybrid space characterized as DigiPlace (Zook and Graham, 2007).

The advances in computer technologies and in new sensor technologies, automated controls, and data telemetry allow for unprecedented development of RTRM in almost all scientific fields such as in meteorology, oceanology, medicine, physics and chemistry, astronomy, environment, and finally of course in detection representation and analysis of all scales of space (architectural, urban and regional planning) which is presented below.

\section{Spatial real-time remote monitoring systems - related works:-}

Today, spatial real-time remote monitoring systems (SRTRM) using imagery from satellite and airborne platforms provide digital data in various scales of spatial observation which determine fields of applications. The most common fields concern:

1. Urban land cover,

2. Land change,

3. urban land sprawl,

4. GPS to GIS applications,

5. room environment and

6. spatial growth-analysis.

The SRTRM take also into account various mapping criteria for characterizing anthropogenic and natural surfaces based on land-cover and land-use mapping methods, accuracy assessment and integrated spatial analysis methods (Treitz and Rogan, 2004). Spatial resolution was identified as the single most important issue for urban remote sensing (Welch, 1982) although its potential was greatly enhanced in the late 1980s with the launch of the SPOT series of satellites (Baraldi and Parmiggiani, 1990; Treitz et al, 1992). Each new generation of satellite is equipped to collect high-spatial resolution data.

These systems are used in Operational Weather Forecasting Systems (OWFS) and they are commonly integrated within a computer environment to constitute a complete real-time forecasting system (Fast et al, 1995). In addition, many contemporary researches on remote spatial urban simulation are based on the Regional Atmospheric and Modeling System (RAMS), which was created in the early 1980's, as a multipurpose, numerical prediction model that simulates atmospheric phenomena (Cotton et al, 2003).

\section{Urban land-cover and land-use}

Land cover is "the physical materials on the surface of a given parcel of land (e.g. grass, concrete, tarmac, water)," and land use "the human activity that takes place on, or makes use of that land (e.g. residential, commercial, industrial)" (Barnsley et al, 2001). Monitoring of urban settlements via multi-temporal remote sensing imagery has received increasingly greater attention in recent years (Gallego, 2004). Many land cover classification methods have been proposed and applied over the last years (Pal and Mather, 2003) based on good performance, simple operation 
and ease in application (Du et al, 2010). Changes in land cover and vegetation were investigated based on multitemporal remote sensing Landsat TM images.

A hierarchical classifier system, among others, was developed, that uses different feature inputs for specific classes and conducted a classification post-processing approach to improve its accuracy ( $\mathrm{Du}$ et al, 2010). Accuracy assessment is an important feature of land-cover and land-use mapping, not only as a guide to map quality and reliability, but also in understanding thematic uncertainty and its likely implications to the end user (Czaplewski, 2003). Prior to image classification, calibration data must be sampled from appropriate areas, in an appropriate support size (Stehman and Czaplewski, 1998). The steps of the proposed classification method (Du et al, 2010) are:

1. Feature extraction and feature set construction,

2. Spectral statistics of land cover,

3. Selection and validation of feature combination for specific land cover types

4. design and classification based on various classes such as Lake water, forest farmland and Built-up, baresoil, unplanted farmland and

5. Post-classification processing based on statistics and cover land change analysis.

An expert system classification model was constructed using the ERDAS Imagine image-processing software (Stefanov et al, 2001). This expert system was applied in the semiarid Phoenix metropolitan portion of the Central Arizona-Phoenix area, where georeferenced information of remotely sensed data such as land use data, spatial texture, and digital elevation models (DEMs) was used to obtain greater classification accuracy. Logical decision rules were used with various datasets assigning class values to each pixel and the spatial texture of Landsat Thematic Mapper data was calculated. This expert system was constructed to perform postclassification sorting of the initial land cover classification using additional spatial datasets such as texture, land use, water rights, city boundaries, and native reservation boundaries.

In other cases, synthetic aperture radar (SAR) and multi-spectral sensors where used for urban area characterization and monitoring (Gomez-Chova et al, 2006). This use is based, on the one hand, on a multi-spectral image allowing the reconstruction of the energy radiated by the Earth's surface throughout the visible and infrared ranges of the electromagnetic spectrum (Clark, 1999) and, on the other hand, on SAR complex images. SAR images are related to the dielectric properties of the target, being sensitive to microtextures, and because microwave radiation penetrates the soil and canopies depending on frequency and polarization, SAR provides information that may not be obtained from optical sensors (Oliver and Quegan, 1998). The proposed methodology takes the following steps:

1. Pre-processing of optical and SAR data using specific algorithms of images detection taking into account the geo-location information available from Landsat,

2. Feature extraction in urban monitoring between Urban/Non-Urban areas such as SAR images processing creating specific algorithms on filters application on images pixels and Multi-spectral optical data processing and finally

3. Feature selection methods applying a feature selection stage in order to reduce dimensionality problems, (Hughes, 1968).

\section{Urban and architectural land change}

A significant component of urban change detection methods using remote sensing is related to the characterization of both natural and urban ecosystem structure and function at synoptic scales (Treitz and Rogan, 2004). The most widely used change detection methods are post-classification comparison (Alo and Pontius, 2008) and multidate composite image change detection (Jensen, 1996). Research continues to focus on the potential for digital image processing of high resolution imagery for detecting, identifying and mapping areas of rapid change (Longley et al, 2001).

Satellite remote sensing and geographic information systems (GIS) have been widely applied in identifying and analyzing land use and land cover change (Ehlers et al, 1990). The reference data were collected from field surveys. Global positioning systems were used to assist in the identification of field data on images. Large-scale aerial photos and QUICKBIRD images were also employed as reference data for the assessment of classification and accuracy (Wu et al, 2006). 
A variety of techniques used to measure/estimate the area change of the urban form (impervious surfaces), such as digitizing the remote sensing images, remote sensing pattern recognition approaches, such as supervised, unsupervised and knowledge-based expert system approaches (Greenberg and Bradley, 1997).

However, the availability of large amounts of data suitable for urban applications demands the development of adequate algorithms and procedures for their automatic characterization and fusion. These data are:

1. 2D aerial data such as Huperspectral, SAR data, etc., for land cover and use maps, fine details, urban infrastructures, objects and materials, etc.,

2. 3D remote sensing data such as stereophotogrammetry, laser scan data etc., for Digital Terrain Model, virtual city environments, building structures, etc., and

3. 2D satellite data such as panchromatic, multispectral, SAR data, etc for coarse details urban area monitoring, urban infrastructures, etc.,

(Gamba et al, 2005).

\section{Urban land sprawl}

Digital image processing and geographical information system (GIS) have been used to extract the information related to sprawl area of impervious surfaces and their spatial and temporal variability classification of the remotely sensed images obtained from various sensors (Kumar Jat et al, 2008). The convergence of GIS and database management systems has helped in quantifying, monitoring, modelling, and subsequently predicting the urban sprawl phenomenon. Landscape metrics (patchiness and map density) were computed in terms of a spatial phenomenon, in order to quantify the urban form (impervious area). Further, multivariate statistical techniques were used to establish the relationship between the urban sprawl and its causative factors. The steps of the proposed methodology are:

1. satellite images, remote sensing data, topo-sheet and thematic data,

2. image pre-processing of data,

3. image classification,

4. impervious area extraction,

5. derivation of urban sprawl metrics, modelling of urban growth and prediction of urban growth, (Kumar Jat et al, 2008).

Statistical techniques, such as multivariate regression, were used to determine the relationship between the percent impervious area and various urban development parameters such as road density, population density, land use type and size of development units (Lo, 2001). Regression analysis can be undertaken to verify this hypothesis such as the relationship between the increased urban land and the distance to urban center, etc (Wu et al, 2006). This method is based on complex models taking into account stationary transition probabilities, distance coefficients, times, etc.

\section{GPS to GIS applications}

The integration of new technologies, such as radio communication permitting wireless interaction between different devices, communication software, GPS devices and GIS applications, enable real-time monitoring of urban space. The various requirements, needs, resources and functions of common services such as acquisition, communications, GPS, GIS, etc., demand an interoperability and specific solutions with new software technologies. Applications must be able to transform themselves intelligently by incorporating new technologies, developing or extending their capabilities (Hecht, 1998).

An object-oriented distributed information system for mobile automatic location (OODISMAL) provides the basic components to integrate radio communication and real-time data captured by GPS units with GIS components (Muro-Medrano et al, 1999). Data captured in real-time by global positioning system (GPS) units are sent to geographical information system (GIS) applications. The architecture of this system consists of:

1. a radio component server that provides the function of a radio to all computers in the local net,

2. a data acquisition component that uses the radio to extract from received messages the data captured by GPS and

3. the components of the radio and data acquisition integration with GIS application, (Muro-Medrano et al, 1999).

The necessary interoperability between devices and software technologies needs specific interfaces which provides the integration and distribution of functions. A Common Object Request Broker Architecture (CORBA) is a typical example of middleware infrastructure to provide interoperability (Muro-Medrano et al, 1999). The COBRA system consists of: 
1. a radio component server, which offers messages received by the radio connected to a PC,

2. the data acquisition component which works as a client of the radio component to receive data from remote GPS, and as a server that offers location information and finally

3. the simulation component which simulates messages within GIS environment with GPS information from a database through the persistence component and has the responsibility, on the one hand, to provide mechanisms to access manage and exploit information and, on the other hand, to provide GIS visualization capabilities, (Muro-Medrano et al, 1999).

The advantages of such system are:

1. the graphical integration with a digital map of functions of other components,

2. flexible development,

3. the reuse of many components in several applications and

4. configuration of applications in different ways according to the requirements of the client, (Muro-Medrano et al, 1999).

\section{Room environment (internal architecture)}

Monitoring sensors such as humidity sensor, temperature sensor, $\mathrm{CO}_{2}$ sensor, flying dust sensor, etc., was developed also for home environment monitoring system. Surveillance camera or web-camera can transfer home vision to personal PC or Personal Digital Assistant (PDA) using web server and data base (Chung and Oh, 2006). The configuration of a home networking system includes a monitoring or remote control software program, a web broadcast server and a console for home networking which are connected via the Internet. A wireless sensor transmitter based on the console for home networking and web cameras connected to the web broadcast server ensure the transmission of moving image and sensor data monitoring to PC or PDA devices (Chung and Oh, 2006).

\section{Spatial growth-analysis}

For the characterization of urban areas and the analysis of urban spatial growth, many studies have been supported through either an image-to-image comparison or a map-to-map comparison. Spatial growth-analysis is based on spatial change detection from image classifications methods, urban land use/cover classification schemes, land use/cover maps comparison, etc.

The image-based comparison is generally accurate but it suffers from inability to provide detailed information of how various urban land use/cover categories change (Yang and Liu, 2005). The image and data classification is significantly important because it provides timely and inexpensive land use information over large areas for environmental purposes, such as source pollution management, etc (Park and Stenstrom, 2008). In contrast, the mapto-map comparison, or post-classification comparison, has the potentiality to detect the nature of urban land use/cover changes (Jensen, 1996).

Among recent urban change characterization methods and spatial growth-analysis researches is the urban imperviousness method which is defined as a sum of impermeable landscape features that include buildings, roads, parking lots, sidewalks, and other built surfaces (Yang and Liu, 2005). An alternative method for urban spatial growth characterization is the satellite-derived imperviousness index creation. This method is based on high resolution imagery and analyzes the spatio-temporal changing trend in landscape imperviousness as related to urban land use/cover changes and establishes a relationship between imperviousness index change and urban land use/cover change detected through image interpretation (Yang and Liu, 2005). The urban spatial growth analysis, via a final index map, is the result of the following previous steps:

1. Landsat data acquisition and preprocessing

2. high-resolution calibration image selection and processing,

3. imperviousness index estimation, and

4. accuracy assessment, (Yang and Liu, 2005).

In addition, remote sensing data, image analysis systems (IAS) and GIS provide opportunities for integrated analysis of spatial data and product development. The interactions of these components have been described in the following three ways (Wilkinson, 1996):

1. Remote sensing data can be used as input data for analysis within a GIS, 
2. GIS data can provide ancillary data for improved remote sensing data analysis for discrimination of land-cover and land-use classes and

3. The application of remote sensing data and other spatial data within a GIS to combine modelling and analysis.

\section{Methodology:-}

Selected geographical coordinates (positions) which participate in simple or complex geometries such as points, polylines, polygons and surfaces, in relation with corresponding local attributes related to geographical reference, are transmitted from the field towards a modeling and simulation system, such as AutoCAD environment. The positions and their attributes, as descriptive information of captured digital sensor's values, are combined to ASCII sentences according to the AX25 protocol, which is used for data transmission and commonly by modems; those are also transmitted in AutoCAD environment as data packets through a radio network (Wilkinson, 1996) creating automatically new entities. The AX.25 protocol, which is an amateur radio adaptation of the ITU-T X.25 protocol, supports the requirements for amateur satellite communication. The AX.25 packets are sent over the radio links (the Physical layer), over the same $145 \mathrm{MHz}$ (uplink and downlink) VHF band (Addaim et al, 2008).

The principal methodological steps are:

1. simultaneous capturing of geographical coordinates with handheld Global Positioning System (GPS) device and related attributes values with digital sensors (e.g. microphone, thermometer, humidity meter, sound pressure meter, thermometer, etc),

2. data transmission process in real-time with a pair of VHF radios which contain a decoder,

3. data receiving, transformation in adequate format and spatial registration into the system via a laptop PC to which peripheral devices are connected via serial ports and adequate communication protocols,

4. modeling and simulation in $\mathrm{CAD}$ environment via specific developed interfaces and software.

\section{Acquisition and transmission method}

Into the field, a connection system of digital devices ensures the data collection and transmission (Fig. 2). This system is made of a hand-held GPS which acquires the geographic coordinates, one or more digital sensors for taking measurements in place, such as temperature, humidity, sound, etc., and a special VHF radio where the previous devices are connected (Papadimitriou and Kouzeleas, 2005).

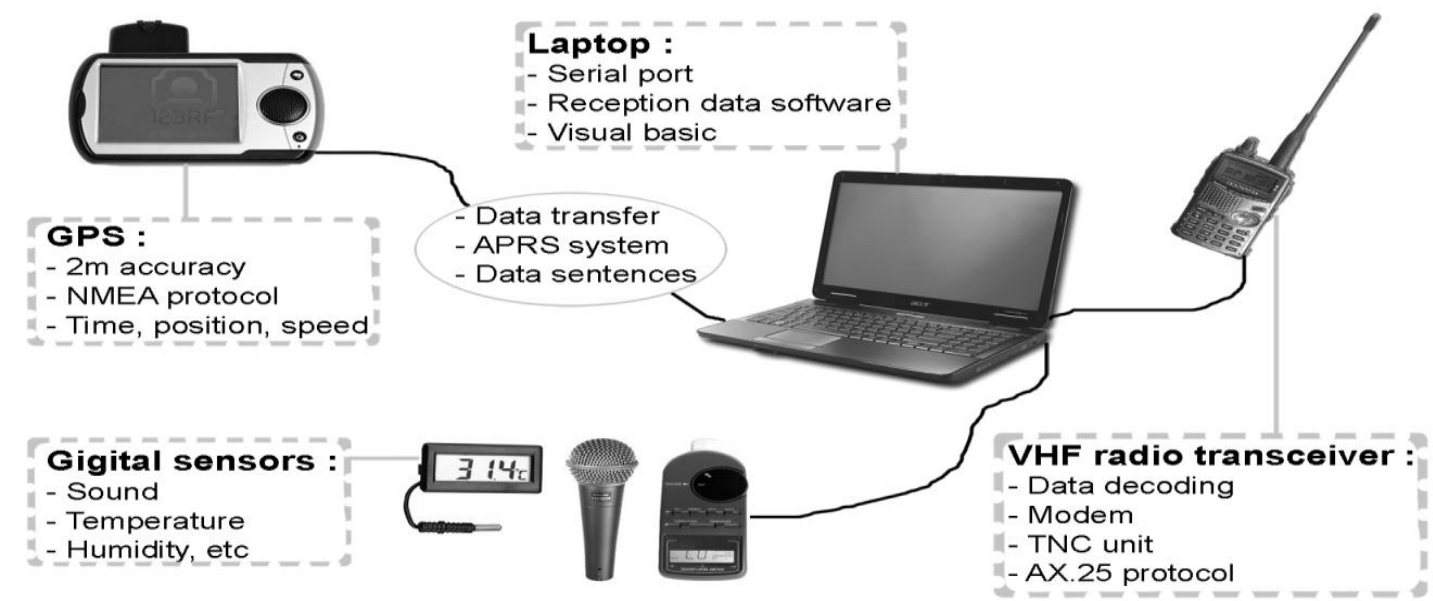

Fig 2:-Connection system of digital devices (hand-held GPS, VHF radio transceiver, digital sensors for measurements) with laptop ensuring remote real-time data acquisition and transmission

The GPS, which provides less than $2 \mathrm{~m}$ accuracy, and the digital sensors, have an output port for data transfer conforming to the National Marine Electronics Association (NMEA) protocol. The NMEA protocol (NMEA, 2010) was used often by GPS-receivers to send their data. These NMEA protocol sentences show the time, position and speed of the device (Eijk and Roeloffs, 2010). The VHF radio, with the aid of a specific decoder, turns the digital sensors measurements into data packets transmitting them as voice signals in VHF frequencies which are readily available and inexpensive (Paffet et al, 1998). These data packets form specific data sentences including positioning and sensors values information according to the syntax for packet radio communication, known as Automatic Position Reporting System (APRS) (Bruninga, 1993) which is very common between radio amateurs. The APRS provides various services such as mobile localization of ships and data collection from autonomous weather stations 
in inaccessible sites and sends telemetry data of the satellite to all amateur ground stations all over the world (Addaim et al, 2008). The transmission of these data sentences is realized with the VHF radio transceiver that contains a special unit known as Terminal Node Controller (TNC). This last unit is used for digital data communication. It organises data in packets according to Amateur Packet AX.25 protocol and provides the modulation/demodulation function for interfacing with transmitter and receiver (Santoni, 2004).

\section{Reception and transformation method}

In an office or mobile station, another similar telecommunication system ensures the data reception and transformation. This system is made of another VHF radio receiver with decoder of the voice signal in the same frequency which is connected to a PC via serial port (Fig. 3). The data reception and transformation process is based on the principles of APRS system. The decoding transmission and the receiving data are supported by software interfaces developed in Visual Basic programming language. Every data sentence creates finally a new record in a data base file containing the:

1. record number,

2. GPS geographical coordinates,

3. GPS time and

4. measurement value,

(Papadimitriou, 2004).

The file is updated automatically with the new data reception. This means maintains a low operational cost even for small projects. Furthermore, the information of all stored records generate, in real-time, dynamic vector entities and thematic maps in AutoCAD environment via a specific software developed in visual LISP and visual BASIC language.

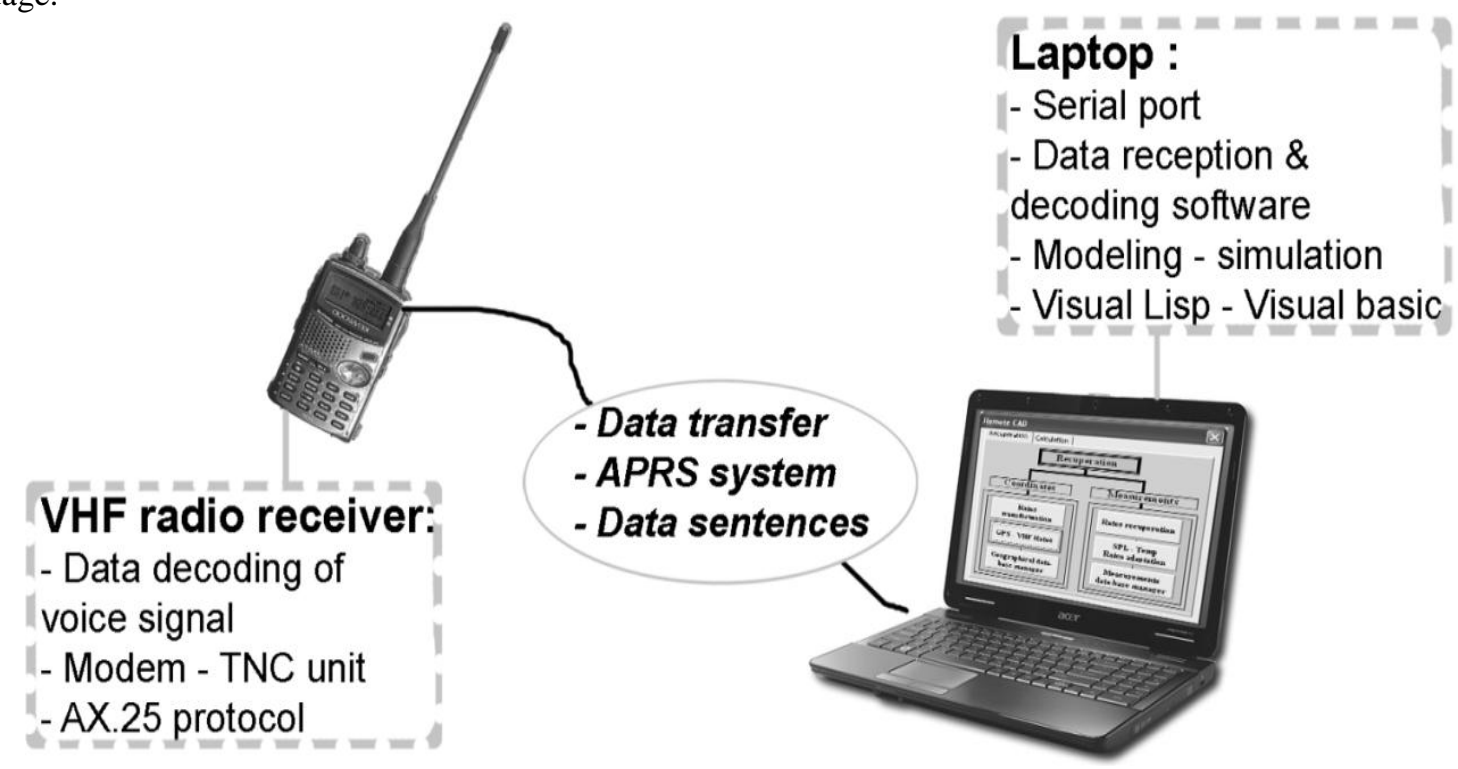

Fig 3:-Connection system of VHF radio receiver with laptop ensuring remote real-time data reception and transformation for modelling and simulation

An alternative solution is the use of individual hand-made connection of a simple transmitter, a specific decoder device and a receiver which are in a small electronic plaque format almost 10X15cm (Papadimitriou and Kouzeleas, 2005) and are distributed only in Internet (Fig. 4) (Tsirtsimpasis, 2003). 


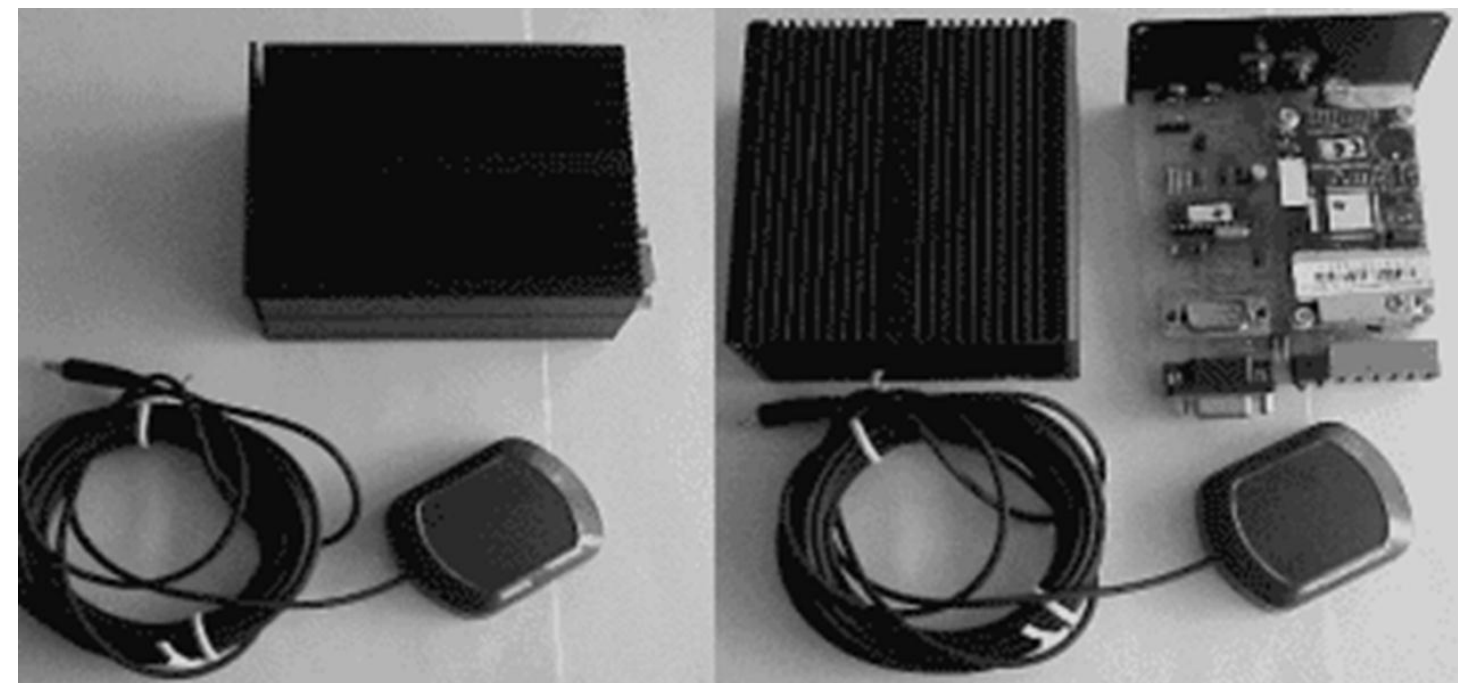

Fig 4:-individual hand-made connection example for the data field transmission which is distributed on the internet. the gps antenna is located externally for better receiving signal (tsirtsimpasis, 2003)

\section{Modeling and simulation process:-}

The participation of the transmitted data in the model parametricism by creating 3D entities in real-time is realized with the aid of specifically developed modeling and simulation add-on software in AutoCAD environment. This software is developed basically in Visual LISP programming language concerning the entities geometry manipulation with specific methodology and adaptation techniques tool in CAD system (Kouzeleas, 2002) and secondly in Visual BASIC database programming language concerning, among other, graphical interface dialogs (Thalheim, 2002). It consists of two complementary components: the "rates recuperation" and the "rates calculation" component (Fig. 5).
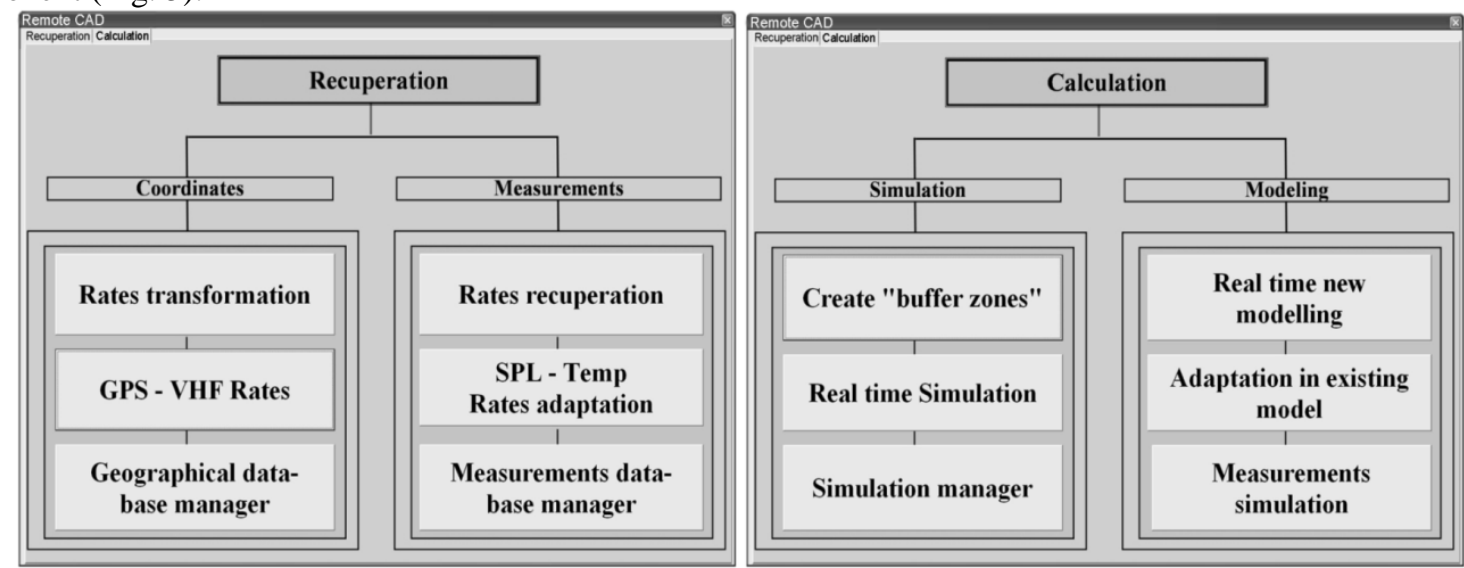

Fig 5:-Rates recuperation and calculation components of specifically developed add-on software in AutoCAD environment handling real-time modelling and simulation processes

The rates recuperation component extracts the absolute geographical coordinate's rates in ASCII format. It defines local coordinates in a pre-existent model in order to adapt the absolute geographical coordinates rates of the field entities in relation with the measurements rates. Specific functions transform the geodetic coordinates into Cartesian coordinates, and every GPS-VHF and measurement rates into an adequate format and stocks these rates in "programming lists" (Kramer, 1997) with the aid of "geographical and measurements data-base managers" developed in Visual LISP. The rates calculation component permits a real-time (automatic) or a momentary (manual) modelling of the transmitted coordinates. The modelling of the new 3D entities can be adapted to the existing environment or can be generated in an "empty" environment. This is achieved by the use of the general forms of transformations of translating, rotating, rescaling (Cromley, 1992), without altering the desired geometrical properties of the entities (Goodchild and Kemp, 1990; Seggern von, 1990). The specific function of the proposed tool defines distances concerning: 
1. serial displacement of the transmitter in urban environment (e.g. human displacement with geographic coordinates registration mobile system, with CAD system and digital sensor device for measurements capture) and

2. selected positions in random displacement in physical environment (e.g. sampling digital registration where there are not any obstacles for the better spatial approach of ecologic interest from GPS).

3. During the modeling process, a function of complex entities simplification is necessary, concerning especially:

4. big number of vertex describing polylines and urban outlines forms (e.g. roads, paths, rivers, railroad, etc) and change scale necessity based on specific algorithms (e.g. "lines generalisation"), (Li, 2007) and

5. triangularisation surfaces problems for location and displacement points, surfaces management, etc, (Chiu and Wang, 2003).

In addition, we can create, in different overlay thematic layers, corresponding measurement simulations based on captured sensor rates with geographical references. Finally, every feature created in real-time can directly participate in GIS processes for further treatment and spatial analysis.

The function process of the proposed system starts with the recuperation of the transmitted data (Fig. 7). These data are stocked in a data-base, calculation sheet or text format. Visual LISP routines get to recuperate these data in a continuous way and stock them in "programming lists". Via the geographic and measurements data-base manager, the geographic coordinates data are transformed in Cartesian coordinates, the GPS time and the measurements data are extracted and adapted into an adequate local coordinate system according to the current design scale. The scale is related to entities simplification which is necessary in order, on the one hand, to transform the transmitted data into models and adapt them via a specific modelling process into the existing environment or be generated in an "empty" modelling environment (Kouzeleas, 2004) and on the other hand, to adapt outlines forms with thousands of lines into more simply forms during the scale change ("line generalisation process"), with almost minus impact to change area. During the real-time modelling, rates measurements, which are stocked in "lists", concern a predefined area. These rates are classified according to their nature (eg. sound, temperature, humidity, etc.) properties, range, their relation to other factors (e.g. sound pressure level in relation to human, biologic and geophysical sounds, etc.) (Papadimitriou and Kouzeleas, 2007), urban build-up area complexity, etc., and they create real-time superposed thematic layers cartography to the modelling environment with geographic reference (fig. 6).
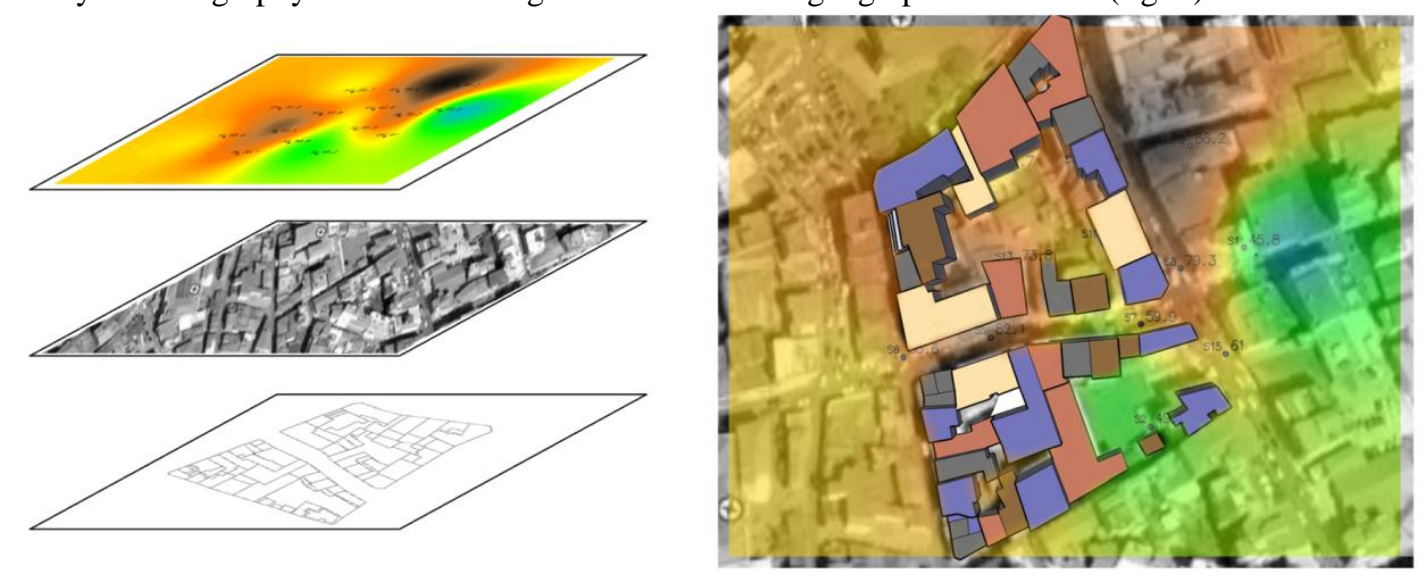

Fig 6:-Superposed thematic layers cartography with real-time modelling of outlines forms in geographic reference and adaptation with satellite image and rates measurements.

The rates, continuously retrieved in real time, feed the system with new data concerning concrete spatial coordinates and rates and the intermediate positions are generated through the interpolation method (Gregory, 2002). The measurements concern concrete spatial location points and the rates for the intermediate positions derive from intercalation and so on. These intermediate rates are refreshed and re-identified with new reception according to the reception cadence. This process permits the redefinition of the model through a new adapted simulation in a timing changeable surface format. Every measurement rate in each position covers a distinguished area where the rates are "diffused" in a quite similar level including a specific range and creating "buffer zones". The accurate examination of these spatial intersections produces a reliable "georeferenced map" with superposed geographic features and physical properties measurements (fig. 7). 


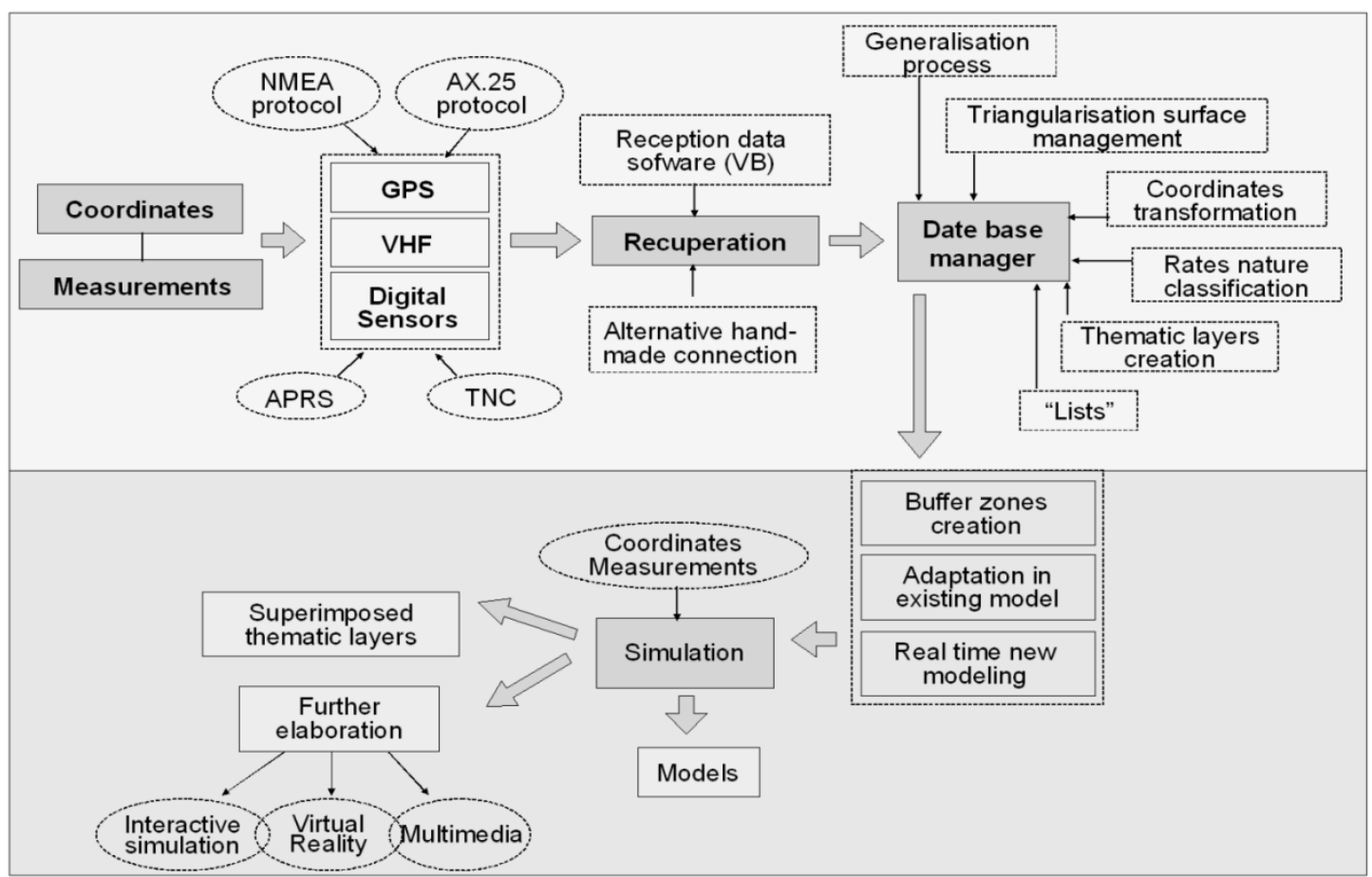

Fig 7:-Function process of proposed remote real-time urban digital design and spatial simultaneous simulation The modelling geometry and the measurements rates can be used for further elaboration in a no-real-time interactive simulation and visualization process of rates describing characteristics of the natural space with geographical reference via geographical coordinates of satellite images of urban and natural environment using virtual reality environment and multimedia components (Kouzeleas, 2008).

\section{Case study: Remote real-time modelling and simulation of Diachronic Museum of Larissa:- The area}

The proposed remote real-time digital design and spatial simultaneous simulation was applied at the 'Mezourlo' hill on the southern outskirts of the city of Larissa, Greece, on an area of 54 acres where the diachronic museum of Larissa is located. The exhibits come from excavations that took place from the 20th century until today at the wider region of Thessaly, as well as from traditions and donations of objects and repatriation of antiquities. A total quantity of about 2,000 items will be exhibited at the exhibition (Odysseus, 2018). The space was chosen as a case study as it combines urban and architectural areas, relative altitude to create Digital Terrain Model (DTM) through 3D mesh modelling, and built space to create a basic perimeter 3D model (Fig. 8).

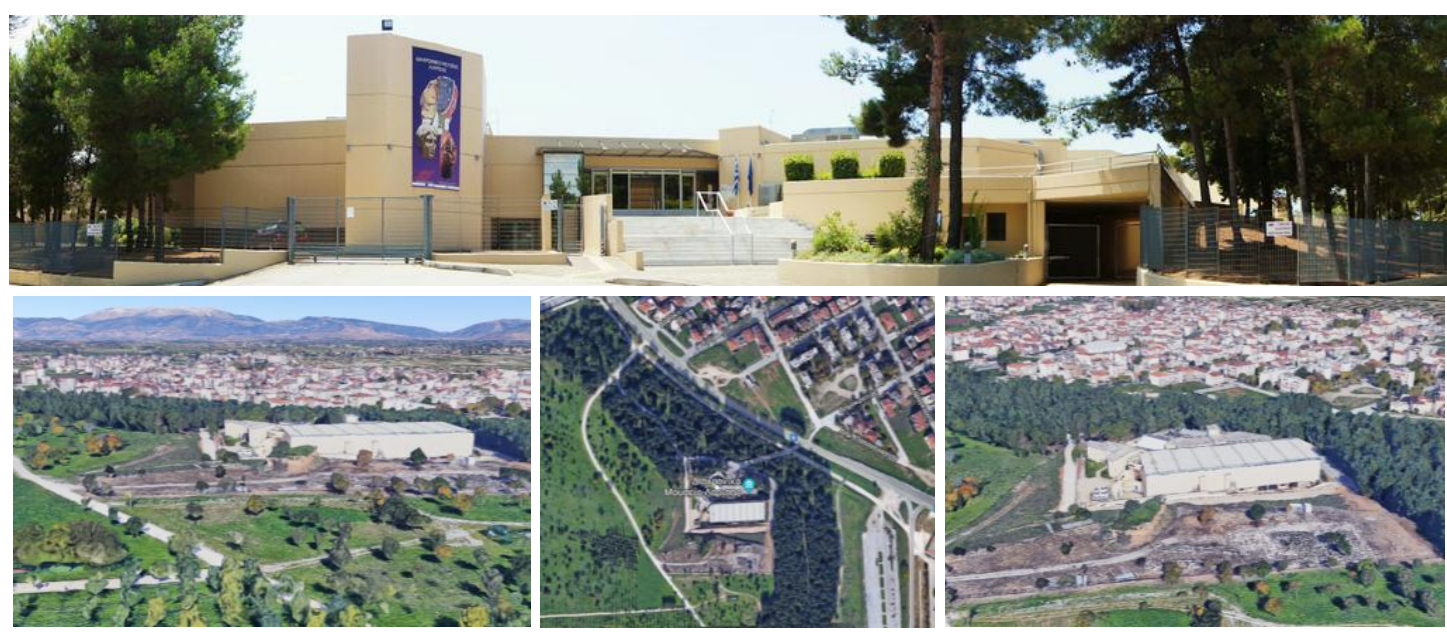

Fig 8:-Views of the Diachronic Museum of Larissa at the 'Mezourlo' hill -City of Larissa - Greece 


\section{Data collection}

Appropriate equipment based on the proposed methodology was used for data collection:

1. PC laptop with intel core i7 Processor

2. GPS antenna of $2 \mathrm{~m}$ accuracy with NMEA protocol

3. Two VHF radio transceivers with modem (TNC units, A.X. 25 protocol)

4. Sound Pressure Level (SPL) digital sensor (SPL-Lab Next-LCD 1 Sensor Kit)

5. Special developed software interface for data and sentences transfer using APRS system

6. Special developed software (CAD remote) for handling real-time modelling / simulation data

Walking around the case study area and having mobile equipment, the user retrieves and sends the GPS position (x, $\mathrm{y}, \mathrm{z})$ and the corresponding SPL value in remote real-time through a digital sensor to the appropriate simulation program for each selected point. The GPS coordinates were retrieved as 3D geodetic coordinates $(\varphi, \lambda, h)$ and translated from the proposed design / simulation program as 3D cartesian coordinates (x, y, z) (Kouzeleas, 2007). The random points refer to positions describing (a) the relief of the area (positions with or no elevation) for 3D mesh creation, (b) the superimposed SPL thematic sound map at exactly the same GPS positions and (c) the contours of the built spaces to create simple perimeter 3D models. The following table accurately describes the positions of the selected points (x, y, z) and the corresponding SPL values (Table 1).

Table 1:-List of points positions and SPL rates

\begin{tabular}{|r|r|r|r|r|r|r|r|r|r|r|r|r|r|r|r|r|}
\hline No & POINT_X & POINT_Y & POINT_Z & SPL & No & POINT_X & POINT_Y & POINT_Z SPL & No & POINT_X & POINT_Y & POINT_Z & SPL \\
\hline $\mathbf{1}$ & 361827,6 & 4386277,0 & 109,7 & $\mathbf{7 1}$ & $\mathbf{1 3}$ & 362122,4 & 4386300,0 & 89,0 & & $\mathbf{2 4}$ & 361892,0 & 4386299,5 & 110,4 & 72 \\
\hline $\mathbf{2}$ & 361815,3 & 4386229,2 & 108,8 & & $\mathbf{1 4}$ & 362118,5 & 4386377,8 & 86,8 & 85 & $\mathbf{2 5}$ & 361966,6 & 4386270,9 & 110,5 & 73 \\
\hline $\mathbf{3}$ & 361815,1 & 4386188,1 & 109,6 & $\mathbf{7 0}$ & $\mathbf{1 5}$ & 362042,3 & 4386419,1 & 89,0 & & $\mathbf{2 6}$ & 362069,7 & 4386242,4 & 98,2 & 72 \\
\hline $\mathbf{4}$ & 361866,1 & 4386227,0 & 110,2 & & $\mathbf{1 6}$ & 361953,4 & 4386470,7 & 86,5 & 86 & $\mathbf{2 7}$ & 361888,5 & 4386409,8 & 98,3 & 76 \\
\hline $\mathbf{5}$ & 361889,9 & 4386188,6 & 110,5 & & $\mathbf{1 7}$ & 361893,8 & 4386516,7 & 84,9 & & $\mathbf{2 8}$ & 361866,7 & 4386458,8 & 93,4 & 75 \\
\hline $\mathbf{6}$ & 361925,6 & 4386153,2 & 111,2 & $\mathbf{7 1}$ & $\mathbf{1 8}$ & 361827,2 & 4386574,7 & 79,4 & 83 & $\mathbf{2 9}$ & 361625,8 & 4386302,9 & 103,1 & 74 \\
\hline $\mathbf{7}$ & 361964,5 & 4386133,3 & 110,6 & & $\mathbf{1 9}$ & 361786,7 & 4386536,6 & 86,6 & & $\mathbf{3 0}$ & 361808,4 & 4386004,4 & 109,6 & 68 \\
\hline $\mathbf{8}$ & 362005,0 & 4386119,8 & 110,2 & & $\mathbf{2 0}$ & 361743,8 & 4386486,5 & 96,0 & 75 & $\mathbf{3 1}$ & 362098,6 & 4386018,2 & 95,2 & 78 \\
\hline $\mathbf{9}$ & 362050,2 & 4386098,4 & 106,9 & $\mathbf{7 4}$ & $\mathbf{2 1}$ & 361747,8 & 4386432,6 & 100,7 & & $\mathbf{3 2}$ & 362237,6 & 4386332,2 & 79,5 & 88 \\
\hline $\mathbf{1 0}$ & 362083,6 & 4386121,4 & 103,8 & & $\mathbf{2 2}$ & 361776,4 & 4386379,4 & 101,5 & 72 & $\mathbf{3 3}$ & 362151,9 & 4386540,9 & 80,9 & 80 \\
\hline $\mathbf{1 1}$ & 362114,5 & 4386161,9 & 98,7 & & $\mathbf{2 3}$ & 361804,2 & 4386334,1 & 107,5 & & $\mathbf{3 4}$ & 361989,7 & 4386652,3 & 80,0 & 75 \\
\hline $\mathbf{1 2}$ & 362119,3 & 4386231,8 & 89,1 & & & & & & & $\mathbf{3 5}$ & 361763,6 & 4386666,5 & 79,4 & 84 \\
\hline
\end{tabular}

The map positions of the points and their respective SPL values are shown schematically in the following pictures. The right map is completed with additional selected points (Fig. 9).
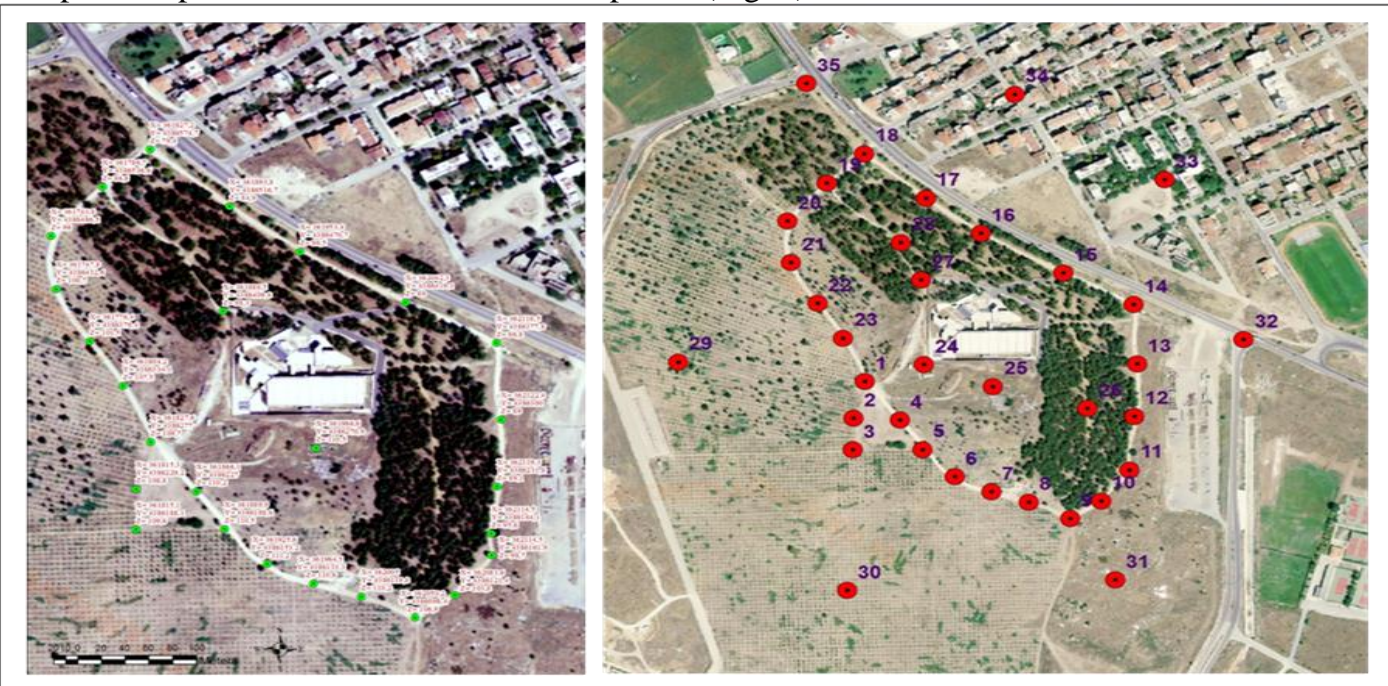

Fig 9:-. Cartographic locations of selected points in the case study area 
The following figure shows schematically the proposed methodology process of

1. points selection in the field,

2. data retrieval and simulation,

3. 3D mesh creation and SPL mapping, and

4. final 3D digital design and spatial simultaneous simulation (Fig. 10).

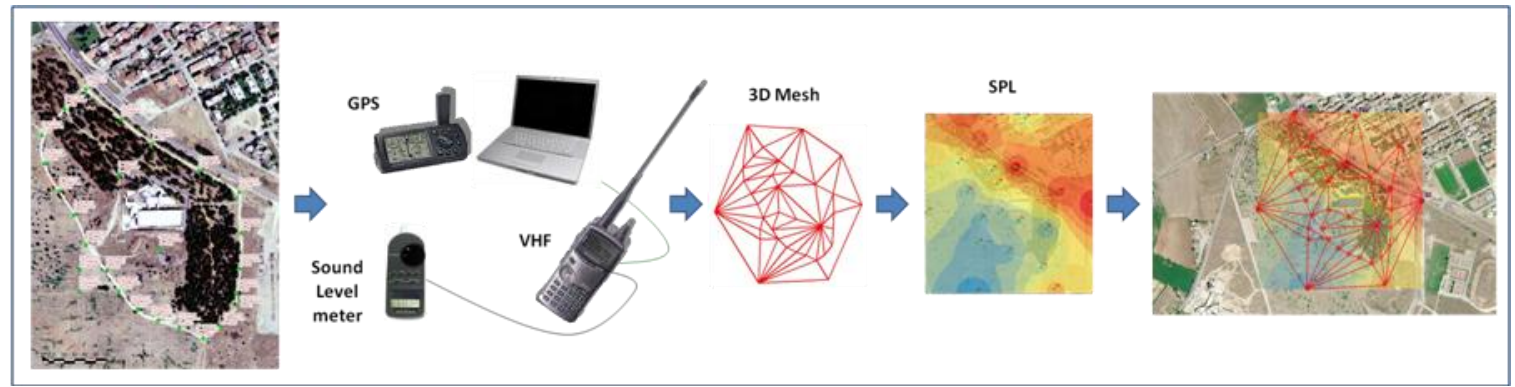

Fig 10:-Schematic representation of 3D digital design and spatial simultaneous simulation process

The proposed methodology, the modelling / simulation process and software allow in the first phase the retrieval and remote sending of data and the 3D mesh digital design of all selected points (physical and building environments) in a CAD environment. The created 3D relief (DTM) is designed as a superimposed layer on an already existing satellite image of the area which was previously adapted to the CAD environment by georeferencing techniques (Hackeloeer et al., 2014). Then the SPL values were imported in the form of an appropriate file in a GIS program (ARC GIS) which creates color thematic mapping using the technique of GIS interpolation (Mitas and Mitasova, 2005). Then the thematic georeferenced map was introduced as a superimposed layer in the CAD environment (Fig. $11)$.

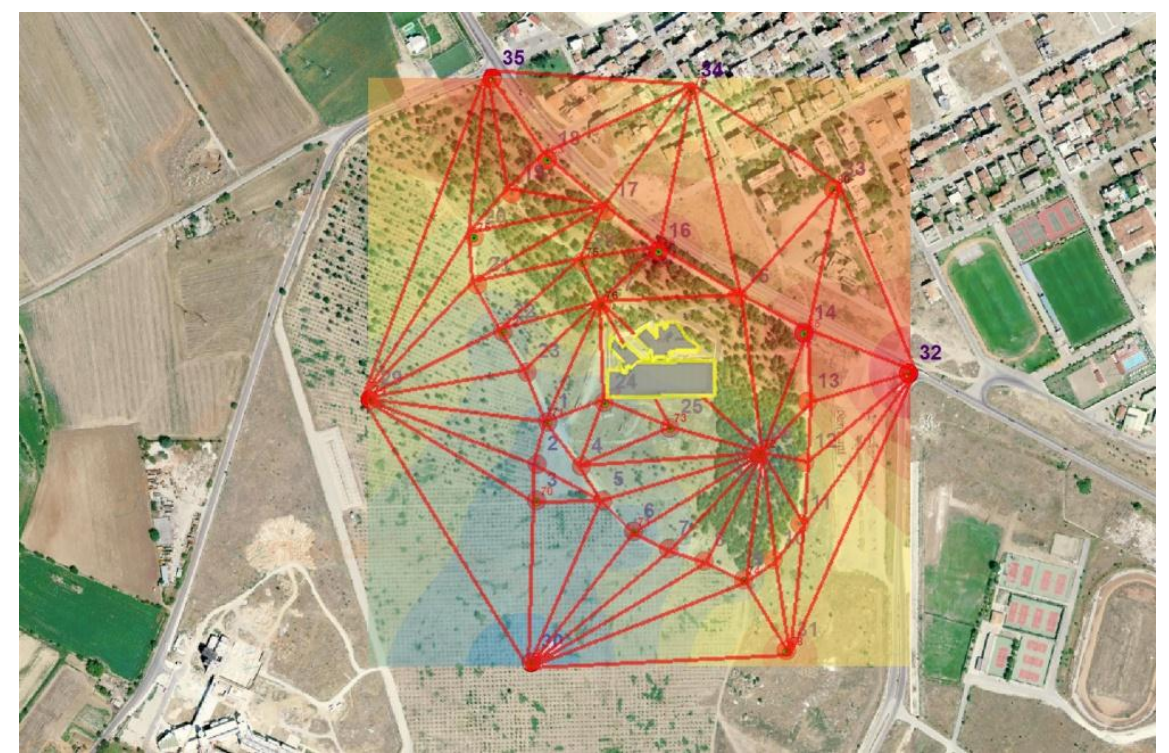

Fig 11:-Final 3D digital design and spatial simultaneous simulation of the case study area

\section{Advantages and disadvantages in comparison with similar technologies:-}

The existing mobile spatial real-time remote monitoring and mapping systems with handheld computers impose limits because of their energy dependence, especially in case of walking users with laptops. In comparison, the proposed system requires less equipment in the field (minimal volume and weight) and it is simpler in use having almost no operational cost.

The existing systems that involve portable computers such as laptops, palmtops or handhelds have more energy autonomy than the basic devices (VHF radio and handheld GPS) which have been selected, but the proposed combination covers efficiently basic requirements, is very simple in use and the energy autonomy of 12-24 hours is enough. 
A major issue is GPS capability in very dense urban areas with high buildings or interiors. A disadvantage of the proposed method is the inefficiency of VHF radio communication in urban areas. The accuracy of handheld GPS devices indicates that the described method can be used for cartographic scales less than 1:10,000 (medium to large scale analysis). Keeping also in mind that VHF communications are preferable in rural and suburb areas (described by cartographic scales 1:10000 and above), it is recommended that such a system should be operated in such cases. In more demanding cases, the overall performance can be improved using combinations of telecommunication means (Internet and cellular phones), mobile phone networks employing processes derived from signal analysis in order to identify and extract recurring patterns of mobile phone for a more detailed understanding of spaces (Reades et al, 2009) and techniques for better GPS accuracy.

The existing systems use several fixed sensors (e.g. meteorological stations) or portable computers (e.g. mobile mapping). In comparison, the proposed system needs only a single sensor that moves around. Regarding the part of data acquisition, it is not necessary to use a computer in the field, as the selected VHF radio manages sufficiently the measurements from the GPS and the sensor.

In many cases of recording with digital means in the existing systems, laptops are used with specific modeling software in real-time. The main problem in such connections is feeding the electrical source, the volume and weight of the devices which are used and the specific software knowledge use. In comparison, the philosophy of the proposed method is that the PC position is not in the field and the field user has the comfort of movement (small weight and volume, enough energy and operational autonomy). The recording can be programmed to be automated regularly or by the user's preference. In addition, when the device is not carried by anybody but it is in a remotecontrolled or free moved means (e.g. meteorological balloon), it can be asked to record remotely in a selected position.

In the advantages of the proposed method, there is the possibility also of data co-elaboration in real-time in modelling environment with adequate programming, either in AutoCAD system, or in other spatial data elaboration and visualization system such as GIS or Internet application.

\section{Conclusions:-}

This method describes the possibility of remote connection of digital devices and sensors in order to record, describe, elaborate, design and visualize spatial data in real-time. The continuous recording of rates leads to 'continuous updating' of a cartographic layer describing simultaneously geographic and other spatial landscape properties in a superimposition.

The remote feed of AutoCAD with spatial data provokes the drawing of new 3D entities (point, linear or area features) in real-time, as the field-user handles a peripheral device of the modelling software (which is connected wirelessly), creating automatically, in this way, the modelling space with a thematic representation of any selected feature and eliminating the time for data transformations (raw data to AutoCAD entities).

This real-time modelling can help architects, city planners, planning development and surveying engineers to model, automatically, in a CAD environment, real objects in architectural scale (squares, buildings position, roads, etc.), or in urban scale such as urban plans or even landscapes (relief, morphology) and finally generate a base of real-time cartographical simulation with 3D geometries enriched with a superposed cartography of spatial properties such as the height of extrusion, sound pressure level, intensity, noise, temperature, etc. This modelling in urban or natural environment can also help to create prototypes of space simulation taking into account parameters such as precise geographical coordinates, noise, natural obstacles of landscape, change of living creatures population (e.g. birds) in order to predict and better handle problems of natural space with ecological dimensions (e.g. vital landscapes, etc). Each entity, which is created in real-time, can directly participate in any spatial analysis operation without further treatment. This could be particularly useful for the dynamic retrieval of spatial information, according to the results of the analysis. This methodology of simulation of space and its properties can constitute a common base of holistic description of space with huge possibilities of development and applications. Using new technologies and multimedia applications provides the opportunity for a more complete approach, description and understanding of external or internal architectural space where access to information and spatial perception can be more 'democratic' and participatory with personal interpretation, understanding and perception of the space from every user. 


\section{References:-}

1. Addaim, A., Kherras, A., and Zantou, E.B. (2008): DSP implementation of integrated store-and-forward APRS payload and OBDH subsystems for low-cost small satellite. Aerospace Science and Technology, 12(4): 308-317.

2. Albrecht, J.H. (1996): Universal GIS operations for environmental modeling. Proceedings of the 3rd International Conference/Workshop on Integrating GIS and Environmental Modeling, Santa Fe, NM, USA.

3. Alo, C.A. and Pontius, Jr. R.G. (2008): Identifying systematic land-cover transitions using remote sensing and GIS: the fate of forests inside and outside protected areas of Southwestern Ghana. Environment and Planning B: Planning and Design, 35(2):280-295.

4. Arvidson, T., Gasch, J. and Goward, S.N. (2001): Landsat 7's long-term acquisition plan - an innovative approach to building a global imagery archive. Remote Sensing of Environment, 78(1-2):13-26.

5. Boulmakoul, A. (2006): Fuzzy graphs modeling for HazMat telegeomonitoring. European Journal of Operational Research, 175(3):1514-1525.

6. Baraldi, A. and Parmiggiani, F. (1990): Urban area classification by multispectral SPOT images. IEEE Transactions on Geoscience and Remote Sensing, 28:674-680.

7. Barnsley, M.J., Møller-Jensen, L. and Barr, S.L. (2001): Inferring urban land use by spatial and structural pattern recognition, Remote Sensing and Urban Analysis, Taylor \& Francis, London, 115-144.

8. Batty, M. (1997): Virtual geography, Futures 29:337-352.

9. Bruninga, B. (1993): A description of APRS by WB4APR, packet users notebook. CQ Magazine, CQ Communication ed, http://www.cq-amateur-radio.com/

10. Chiu, C.S. and Wang, D.I. (2003): Weighted coordinates transformation method for map overlay with nonhomogeneous space partition. Computers \& Geosciences, 29(7):877-883.

11. Chung, W.Y. and Oh, S.J. (2006): Remote monitoring system with wireless sensors module for room environment. Sensors and Actuators B: Chemical, 113(1):64-70.

12. Clark, R.N. (1999): Spectroscopy of Rocks and Minerals, and Principles of Spectroscopy. Manual of remote sensing, John Wiley \& Sons ed., New York.

13. Cotton, W.R., Pielke, R.A., Walko, R.L, Liston. G.E., Tremback, C., Jiang, H., McAnelly, R.L., Harrington, J.Y., Nicholls, M.E., Carrio, G.G. and McFadden, J.P. (2003): RAMS 2001: current status and future directions. Meteorology and Atmospheric Physics, 82:5-29.

14. Cromley, R.G. (1992): Digital Cartography, Prentice-Hall ed., Englewood Cliffs, NJ.

15. Czaplewski, R.L. (2003): Accuracy assessment of maps of forest condition: statistical design and methodological considerations. Remote Sensing of Forest Environments: Concepts and Case Studies, 115-140.

16. Devillers, R., Stein, A. and Bedard, Y.N. (2010): Thirty Years of Research on Spatial Data Quality: Achievements, Failures, and Opportunities. Transactions in GIS, 14(4):387-400.

17. Du, P., Li, X., Cao, W., Luo, Y. and Zhang, H. (2010): Monitoring urban land cover and vegetation change by multitemporal remote sensing information. Mining Science and Technology, 20(6):922-932.

18. Ehlers, M., Jadkowski, M.A., Howard, R.R. and Brostuen, D.E. (1990): Application of SPOT data for regional growth analysis and local planning. Photogrammetric Engineering \& Remote Sensing, 56:175-180.

19. Eijk, V.O. and Roeloffs, M. (2010): Forensic acquisition and analysis of the Random Access Memory of Tom-Tom GPS navigation systems. Digital Investigation, 6(3-4):179-188.

20. Fast, J.D., O'Steen, B.L and Addis, R. P. (1995): Advanced atmospheric modeling for emergency response. Journal of Applied Meteorology, 34:626-649.

21. Gallego, F. J. (2004): Remote sensing and land cover area estimation. International Journal of Remote Sensing, 25:3019-3047.

22. Gamba, P., Dell'Acqua, F. and Dasarathy, B. (2005): Urban remote sensing using multiple data sets Past present and future. Information Fusion, 6(4):319-326.

23. Glasgow, H., Burkholder, J., Reed, R., Lewitus ,A. and Kleinman, J. (2004): Real-time remote monitoring of water quality: a review of current applications, and advancements in sensor, telemetry and computing technologies. Journal of Experimental Marine Biology and Ecology, 300(1-2):409-448.

24. Gomez-Chova, L., Fernandez-Prieto, D., Calpe, J., Soria, E., Vila, J. and Camps-Valls, G. (2006). Urban monitoring using multi-temporal SAR and multi-spectral data. Pattern Recognition Letters, 27(4):234-243.

25. Goni, A., Burgos, A., Dranca, L., Rodriguez, J., Illarramendi, A. and Bermudez, J. (2009): Architecture, cost-model and customization of real-time monitoring systems based on mobile biological sensor data-streams. Computer Methods and Programs in Biomedicine, 96(2):141-157.

26. Goodchild, M.F. and Kemp, K.K. (1990): Introduction to GIS. NCGIA Core Curriculum, University of California, Santa Barbara, CA, pp. 249.

27. Greenberg, J.D. and Bradley, G.A. (1997): Analyzing the urban-wildland interface with GIS. J. Forestry, 95:18-22.

28. Gregory, I.N. (2002): The accuracy of areal interpolation techniques: standardising 19th and 20th century census data to allow long-term comparisons. Computers Environment and Urban Systems, 26(4):293-314. 
29. Hackeloeer, A., Klasing, K., Krisp, J.M. and Meng, L. (2014): Georeferencing: a review of methods and applications. Annals of GIS, 20(1):61-69.

30. Hecht, L. (1998): GIS helps utilities thrive in a deregulated environment. ESRI ArcUser Magazine, http://www.esri.com/news/arcuser/798/utilities.html

31. Hughes, G.F. (1968): On the mean accuracy of statistical pattern recognizers. IEEE Transactions on Information Theory, 14(1):55-63.

32. Jensen, J.R. (1996): Introductory Digital Image Processing: A Remote Sensing Perspective. Prentice Hall, second ed., Upper Saddle River, NJ.

33. Kouzeleas, S. (2002): Development of a help tool in architectural acoustics simulation adaptable in a CAD system. $\mathrm{PhD}$ dissertation, Order number 2642, University of Bordeaux 1, France.

34. Kouzeleas, S. (2004): Computational processes of a hall cad modelisation for acoustic simulation according to accepted geometry format via acoustic software. E-Proceedings of the 1st International Conference-From Scientific Computing to Computational Engineering- (IC-SCCE), 8-10 September, Athens, Greece.

35. Kouzeleas, S. (2007): Conversion of GPS data to cartesian coordinates via an application development adapted to a CAD modelling system. 2nd International Conference on Experiments / Process / System Modeling / Simulation \& Optimization, 2nd IC-EpsMsO, E-Proc, ISBN: 960-530-093-1, University of Patras, 4-7 July 2007, Athens, Greece.

36. Kouzeleas, S. (2008): Methodology of interactive simulation and visualization of acoustic rates of urban space with digital geographic reference. Proceedings of the National Conference of Acoustics, Hellenic Institute of Acoustics, Architecture school of Democritus University of Thrace, Xanthi, Greece, 301-309.

37. Kramer, B. (1997): AutoLISP treasure chest, Miller Freeman Books, San Francisco.

38. Kumar, Jat. M., Garg, P.K. and Khare, D. (2008): Monitoring and modeling of urban sprawl using remote sensing and GIS techniques. International Journal of Applied Earth Observation and Geoinformation, 10(1):26-43.

39. Laurini, R. (2000): A Short Introduction to TeleGeoProcessing and TeleGeoMonitoring. Proceedings of the 2nd International Symposium on Telegeoprocessing, Sophia- Antipolis, France, 1-12.

40. Li, Z. (2007): Algorithmic foundation of multi-scale spatial representation. CRC Press, Taylor \& Francis Group, USA.

41. Lo, C.P. (2001): Modeling the population of China using DMSP operational Linescan system nighttime data. Photogrammetric Engineering \& Remote Sensing, 67:1037-1047.

42. Longley, P.A., Barnsley, M.J. and Donnay, J.P. (2001): Remote sensing and urban analysis: a research agenda. Remote Sensing and Urban Analysis, Taylor and Francis, London, pp. 268.

43. Metternicht, G., Hurni, L. and Gogu, R. (2005): Remote sensing of landslides. An analysis of the potential contribution to geo-spatial systems for hazard assessment in mountainous environments. Remote Sensing of Environment, 98(23):284-303.

44. Mitas, L., Mitasova, H. (2005): Spatial Interpolation. In: Longley, P.A., Goodchild, M.F., Maguire, D.J. and Rhind, D.W., Eds., Geographic Information Systems: Principles, Techniques, Management and Applications, 2nd Edition, $1(2): 34$.

45. Muro-Medrano, P.R., Infante, D., Guillo, J., Zarazaga, J. and Banare, J.A. (1999): A CORBA infrastructure to provide distributed GPS data in real time to GIS applications. Computers Environment and Urban Systems, 23(4):271-285.

46. Netting, R. (2010): Landsat-7. NASA Official, http://science.nasa.gov/missions/landsat-7

47. NMEA, (2010): National Marine Electronics Association, http://www.nmea.org

48. Odysseus, (2018): Diachronic Museum of Larissa, http://odysseus.culture.gr/h/1/eh151.jsp?obj_id=3452

49. OGC, (2010): Open Geospatial Consortium, http://www.opengeospatial.org/

50. Oliver, C. Quegan, S. (1998): Understanding SAR Images, Artech House ed, Boston, MA.

51. Pal, M. and Mather, P.M. (2003): An assessment of the effectiveness of decision tree methods for land cover classification. Remote Sensing of Environment, 86:554-565.

52. Papadimitriou, K. (2004): Location Based Information System for Decision Support in Emergencies. PhD dissertation, Aristotle University of Thessaloniki, Department of Surveying Engineering, Greece.

53. Papadimitriou, K. and Kouzeleas, S. (2005): Description of a system for real-time modelling and simulation of mapping properties. Proceedings of the 1st Greek Congress in Urban Regional Planning and Development, University of Thessaly, Volos 12-14 May, Greece.

54. Papadimitriou, K. and Kouzeleas, S. (2007): Methodological approach of the geographical dimension of urban soundscape. Journal of Geographies, Department of Geography, Charocopio University, Exantas ed, Greece, 13(2):145-157.

55. Paffet, J., Jeans, T.G. and Ward, J. (1998): VHF-band interference avoidance for next generation small satellites. Proceedings of the 12th AIAA/USU Conference on Small Satellites, Logan, Utah, USA.

56. Park, M.H., Stenstrom, K.M. (2008): Classifying environmentally significant urban land uses with satellite imagery. Journal of Environmental Management, 86(1):181-192.

57. Paulson, R.W. (1975): Use of earth satellite technology or telemetry of hydrometerological station data. Proceedings of the International Seminar on Modern Development in Hydrology, Padova, Italy, pp. 1-75. 
58. Reades, J., Calabrese, F. and Ratti C. (2009): Eigenplaces: analysing cities using the space - time structure of the mobile phone network. Environment and Planning B: Planning and Design, 36:824-836.

59. Santoni, F. (2004): Risk management for micro-satellite design. Acta Astronautica, 54(3):221-228.

60. Schneider, W.C. and Garman, A.A. (1981): Tracking and data relay satellite system: NASA's new spacecraft data acquisition system. Acta Astronautica, 8(2):179-194

61. Seggern, D.H.von. (1990): CRC Handbook of Mathematical Curves and Surfaces. CRC Press, Boca Raton, FL, pp. 286.

62. Short, N.M., Lowan, Jr. P.D., Freden, S.C. and Finch, Jr. W.A. (1976): Mission to earth: Landsat views the world. NASA Special Publication 360, Washington, DC: NASA Science and Technical Information Office.

63. Stefanov, W. Ramsey, M. and Christensen, P. (2001): Monitoring urban land cover change: An expert system approach to land cover classification of semiarid to arid urban centers. Remote Sensing of Environment, 77(2):173185.

64. Stehman, S.V. and Czaplewski, R.L. (1998): Design and analysis for thematic map accuracy assessment: fundamental principles. Remote Sensing of the Environment, 64:331-344.

65. Tanzi, T.J. (2000): Principles and Practices in TeleGeomatics. Proceedings of the 2nd International Symposium on Telegeoprocessing, Sophia-Antipolis, France, pp. 123-124.

66. Thalheim, A.M. (2001) : VBA Pour AutoCAD 2002 La référence complète. Thalheim consultants inc, Québec.

67. Treitz, P.M., Howarth, P.J. and Gong, P. (1992): Application of satellite and GIS technologies for land-cover and landuse mapping at the rural-urban fringe: a case study. Photogrammetric Engineering and Remote Sensing, 58:439448.

68. Treitz, P. and Rogan, J. (2004): Remote sensing for mapping and monitoring land-cover and land-use change - an introduction. Progress in Planning, 61(4):269-279.

69. Tsirtsimpasis, S. (2003): SmartAPRS. http://www.houseofradio.gr

70. Turner, J.F. and Woodham, W.M. (1980): Evaluation of remote hydrologic data-acquisition systems. USGS Water Resources Investigations, West-Central Florida, Reston, VA, pp. 79-102.

71. Nature, (2013): Landsat 8 to the rescue. International weekly journal of science, https://www.nature.com/news/landsat8-to-the-rescue-1.12365

72. Weis, M., Müller, S., Liedtke, C.E. and Pahl, M. (2005): A framework for GIS and imagery data fusion in support of cartographic updating. Information Fusion, Fusion of Remotely Sensed Data over Urban Areas, 6(4):311-317.

73. Welch, R. (1982): Spatial resolution requirements for urban studies. International Journal of Remote Sensing, 3:139146.

74. Wilkinson, G.G. (1996): A review of current issues in the integration of GIS and remote sensing data. International Journal of Geographical Information Systems, 19:85-101.

75. Wu, Q., Li, H.Q., Wang, R.S., Paulussen, J., He, Y., Wang, M., Wang, B,H. and Wang, Z. (2006): Monitoring and predicting land use change in Beijing using remote sensing and GIS. Landscape and Urban Planning, 78(4):322-333.

76. Wulder, M. White, J., Goward, S., Masek, J., Irons, J., Herold, M., Cohen, W., Loveland, Th. and Woodcock, C.E. (2008): Landsat continuity: Issues and opportunities for land cover monitoring. Remote Sensing of Environment, 112(3):955-969.

77. Xu, W., Wooster, M.J., Roberts, G. and Freeborn, P. (2010): New GOES imager algorithms for cloud and active fire detection and fire radiative power assessment across North, South and Central America. Remote Sensing of Environment, 114(9):1876-1895.

78. Yang, X. and Liu, X. (2005): Use of satellite-derived landscape imperviousness index to characterize urban spatial growth. Computers, Environment and Urban Systems, 29(5):524-540.

79. Zook, M.A. and Graham, M. (2007): Mapping DigiPlace: geocoded Internet data and the representation of place. Environment and Planning B: Planning and Design, 34(3):466 - 482. 